\title{
Relative numerosity as a dimension of stimulus control: The peak shift
}

\author{
W. K. HONIG and KAREN E. STEWART \\ Dalhousie University, Halifax, Nova Scotia, Canada
}

\begin{abstract}
Pigeons were trained to discriminate between arrays containing equal numbers of two different elements as $\mathbf{S}+$, and arrays which contained more elements of one kind than the other as $\mathrm{S}-$. They were then tested with the full range of the proportions of the two elements. This resulted in behavioral contrast and peak shift, as the pigeons responded more to arrays containing more positive than negative elements than they did to the positive training arrays. These findings were obtained with elements that differed in color (blue vs. red dots) and with elements that differed in orientation (horizontal vs. vertical rectangles). The results indicate that the stimulus control exerted by the derived dimension of relative numerosity involves the same processes as the fundamental dimensions that characterize simple stimulus elements.
\end{abstract}

Research in the area of stimulus control is mostly carried out with simple stimuli in order to study those aspects of stimulus generalization and discrimination that are governed by dimensional aspects of the stimuli. Guttman and Kalish (1956) presented pigeons with spectral values on a response key, while Honig, Boneau, Burstein, and Pennypacker (1963) displayed a line in various orientations. Jenkins and Harrison (1960) used pure tones that differed in frequency. Such simple stimuli presumably facilitate the study of their dimensional aspects, and they permit psychologists to study the effects of discrimination training. One such effect is the peak shift. The subject discriminates two rather similar values presented successively and is then tested with a range of stimulus values. Frequently, more responses are emitted to values that lie beyond $\mathrm{S}+$ (in relation to $\mathrm{S}-$ ) than to the positive training value. This peak shift was originally obtained with pigeons on the spectral dimension by Hanson (1959), Honig (1962), and Honig, Thomas, and Guttman (1959), and it has been replicated many times thereafter (see Honig \& Urcuioli, 1981, and Rilling, 1977, for reviews). Even when a peak shift is not obtained after discrimination training, subjects respond more to stimulus values beyond $\mathrm{S}+$ than they would if they were trained only with $S+$. This is sometimes known as an area shift, because the area under the generalization function appears to have shifted away from the negative training value.

Properties of complex arrays of stimulus elements can exert stimulus control in ways that are similar to the control exercised by individual elements. Honig and Stewart

This research was supported by Grant AO-104 from the Natural Sciences and Engineering Research Council of Canada. William Hayes provided the computer programs for the experimental procedures and data analysis. Correspondence should be addressed to W. K. Honig, Psychology Department, Dalhousie University, Halifax, NS, Canada B3H $4 \mathrm{~J} 1$.
(1989) obtained discrimination gradients based on relative numerosity. Pigeons were trained to discriminate between two uniform arrays of elements. For example, a uniform array of blue dots was associated with reinforcement, whereas a similar array of red dots was associated with extinction. When the pigeons were tested with various proportions of the positive and negative elements, orderly gradients of responding reflected the proportional values. This finding was obtained with elements that differed in color, form, size, and conceptual category. When the total numbers of elements were changed, the discrimination transferred well on the basis of the relative proportions of the elements. Similar results have been obtained from maintained discriminations, in which one uniform array is positive, and all other proportions of elements are presented as negative training arrays.

If the relative numbers of elements constitute a functional stimulus dimension, we should be able to replicate the peak shift, or area shift, with discrimination gradients obtained on that dimension. This was the purpose of the present research. The positive training arrays represented the middle value on that dimension: equal numbers of red and blue dots in Experiments 1A-1C and 2, and horizontal and vertical rectangles in Experiment 3. The negative training arrays consisted of more of one kind of element than the other-for example, more blue than red elements. After the birds learned the discriminations, they were tested with the full range of proportions, in order to determine whether a peak shift would be obtained among the arrays that contained relatively few negative elements. For example, if the positive arrays $(\mathrm{S}+)$ consist of equal numbers of blue and red elements, and if one or more arrays with more red than blue elements are negative $(\mathrm{S}-$ ), then the pigeons may respond more in a postdiscrimination test to arrays containing fewer red than blue element than they do to $\mathrm{S}+$. This would be a peak shift on the dimension of relative numerosity. 


\section{EXPERIMENT 1A Initial Discrimination Training}

Pigeons were first trained to respond to arrays of 18 red and 18 blue elements as $S+$. Then they were tested with the full range of proportions of elements to obtain a "reference" gradient prior to discrimination training. Discrimination training was then carried out between $\mathrm{S}+$ and arrays of 25 blue and 11 red elements (or the reverse) as $\mathbf{S}-$. The birds did not acquire this discrimination, so it was made easier by contrasting the $S+$ arrays with uniform arrays of blue (or red) dots as $\mathbf{S}-$. They did learn this discrimination, and were tested with the full range of stimulus values. Then they were retrained on the more difficult discrimination and tested again. In the last phase, transfer of the discrimination was tested with arrays of 64 elements.

\section{Method}

Subjects. The subjects were initially 4 mature White Carneau pigeons. Prior research experience had not included any tasks related to the present work. One subject responded at such a low rate that the postdiscrimination gradients were erratic and unreliable, and this bird was replaced by a 5 th pigeon. The pigeons were maintained at $80 \%$ of their free-feeding weights.

Apparatus and Stimulus patterns. The training and test chambers and the stimulus patterns were the same as those used by Honig and Stewart (1989) and Honig (1991b). The patterns were backprojected onto a translucent response panel, $7.5 \mathrm{~cm}$ square. Mixed grain was presented from a hopper located directly below the response panel. The hopper was raised for $4.0 \mathrm{sec}$ to provide reinforcement.

The training patterns consisted of 36 round elements, presented as a square matrix in the following proportions: 36 red, 0 blue (1.00); 30 red, 6 blue $(.83) ; 25$ red, 11 blue $(.69) ; 21$ red, 15 blue (.58); 18 red, 18 blue $(.50) ; 15$ red, 21 blue $(.42) ; 11$ red, 25 blue (.31); 6 red, 30 blue (.17); 0 red, 36 blue $(.00)$. The elements were $4 \mathrm{~mm}$ in diameter. The distance between adjacent elements was $11 \mathrm{~mm}$, center to center. The different proportions were presented in several different randomized arrangements. Each slide, being square, could also be presented in various orientations. This provided numerous specific patterns for each proportion of elements. Sample patterns are illustrated in Honig and Stewart (1989), Figure 1. Some transfer tests were carried out with arrays of 64 elements (see Experiment $1 \mathrm{C}$ )

To simplify this account, one kind of element will be called positive, and the other negative, even though both elements appeared in most of the training and test arrays. For example, if arrays of equal numbers of red and blue elements were positive, and the arrays with more blue than red elements were negative, then the red elements are identified as "positive," and the blue elements are "negative," even though blue elements were part of the positive array, and some red elements appeared in all but one of the negative arrays.

Training and test procedures. When two proportions of elements were used in training, each training session consisted of 24 trials, half of them positive and half of them negative. Several random orders of positive and negative trials were used, with the restriction that not more than three positive or three negative trials could occur in succession. Each trial lasted at least $20 \mathrm{sec}$. At that time, a negative trial ended without reinforcement. A positive trial ended with the first response after $20 \mathrm{sec}$, which was not counted, but which caused the grain hopper to be elevated for $4 \mathrm{sec}$ so that the bird could eat from it.
Gradient tests were carried out in extinction. Each test session comprised four blocks of trials. Two test sessions were carried out on consecutive days. Each trial ended after $20 \mathrm{sec}$. The test patterns were composed of the nine proportions of elements listed above. Each proportion was presented once in each block of trials in a randomized order. In the transfer arrays, the 64 elements were 3 rather than $4 \mathrm{~mm}$ in diameter on the response screen, and they were spaced $8 \mathrm{~mm}$ apart, center to center, rather than $11 \mathrm{~mm}$. The proportions of elements corresponded closely to those used for the 36-element arrays.

Measures of discrimination and generalization. For each training session, the total responses to the positive arrays were divided by the total responses to the positive and negative arrays to provide a discrimination ratio (DR). This corrects for differences in the absolute response levels between subjects.

Data from generalization tests are presented as means of total responses to each test value. Since the total response output differed between birds during testing, we provide indices of the slopes of the gradients for individual subjects as summary measures. The number of responses to each test stimulus was expressed as a proportion of the responses obtained with $S+$, which was always the array with $50 \%$ of each kind of element. The four proportions on the "negative" side of $S+$ were averaged to provide a negative slope index, or negative $S I$. If discrimination training is effective, the negative $S I$ is less than 1.0 and can approach zero. The four proportions on the "positive" side of S+ were averaged to provide a positive slope index, or positive $S I$. This value could also be less than 1.0, because of generalization decrement. However, if the trained discrimination produces a peak shift, or a general enhancement of responding among the positive values, the positive SI will increase, and it may exceed 1.0. These indices are presented in the tables, which also provide an index of the highest level of responding obtained from a test value on the positive side of the gradient (the maximum value, or Max, which can indicate a peak shift), and the lowest level of responding on the negative side (the minimum value, or Min). The total numbers of responses obtained from each test are also shown in these tables.

Order of treatments. The birds were first trained to eat reliably out of the grain magazine in the course of one or two training sessions, during which grain was presented, together with the feeder light, at irregular intervals. Then they were trained to peck at the positive arrays containing 18 red and 18 blue elements. Each session consisted of 28 positive trials. A postacquisition test was then carried out for two sessions, with the nine proportions of red and blue elements, in order to obtain a generalization gradient following acquisition of responding with a single positive training value.

After this first test, the birds were trained to discriminate between patterns containing 18 elements of each kind as the positive arrays $(\mathrm{S}+)$ and patterns of 11 positive elements and 25 negative elements as the negative arrays $(\mathrm{S}-$ ). For 2 subjects, the patterns with more red elements were negative; for the other 2 birds, the patterns containing more blue elements were negative.

This discrimination proved to be too difficult. After 10 sessions, the difference between the positive and negative arrays was increased. The arrays with equal numbers of blue and red elements remained positive, but the negative patterns were the uniform arrays of 36 blue or red elements.

This change facilitated the discrimination. Every bird attained a DR of .90 or more during three out of four consecutive sessions. Each subject was then given a postdiscrimination test identical to the postacquisition test. The pigeons were then retrained on the difficult discrimination and were tested two more times. The third test followed this retraining phase, and it involved the same proportions of 36 elements used previously. The fourth test was a transfer test with the patterns of 64 red and blue elements. The individual subjects received various numbers of training sessions between 
tests and acquired the difficult discrimination to various degrees. These matters are described with the results.

\section{Results}

Responding to the positive array was stable after 10 sessions of acquisition training. The total responses emitted during the 10th session ranged from 1,109 to 1,571 for the 4 birds. Figure 1 shows the mean total responses to the different proportions of elements obtained in testing. The first test provided a modest decremental gradient to either side of the training value. However, the mean gradient does not reflect the variability both in total test responding and in the slopes of the individual gradients. For example, Subject 4947 emitted 824 responses during the test sessions and actually responded more to both of the uniform arrays than to the training array. Subject 232 emitted 2,043 responses during the test, 391 of them to the training array, only 102 to the uniform red arrays, and none to the all-blue array. The slope indices are shown for each subject in Table 1. The negative and positive SIs are identified in terms of the subsequent procedures-the proportions of elements that would be negative or positive in discrimination training.

As indicated earlier, the birds did not acquire the initial, difficult discrimination well; Subject 4568 reached a DR of .62 after 10 training sessions; none of the other birds exceeded .60 . When the discrimination was then made easier, the birds acquired it at very different rates. Subject 232 never responded at all to the uniform array of blue elements ( $\mathrm{S}-$ ) and was tested after five sessions. Subject 4947, on the other hand, started at the chance level (DR of .46), but discriminated perfectly by the 14th training session. Subject 4181 started with a DR of .56 and ended with a consistent level of performance at .90 during the sixth block of 5 training sessions. Subject 4568 started with a DR of .84 and was trained for 13 sessions. Thus, 2 birds showed some transfer from the difficult to the easier discrimination. Table 1 provides discrimina-
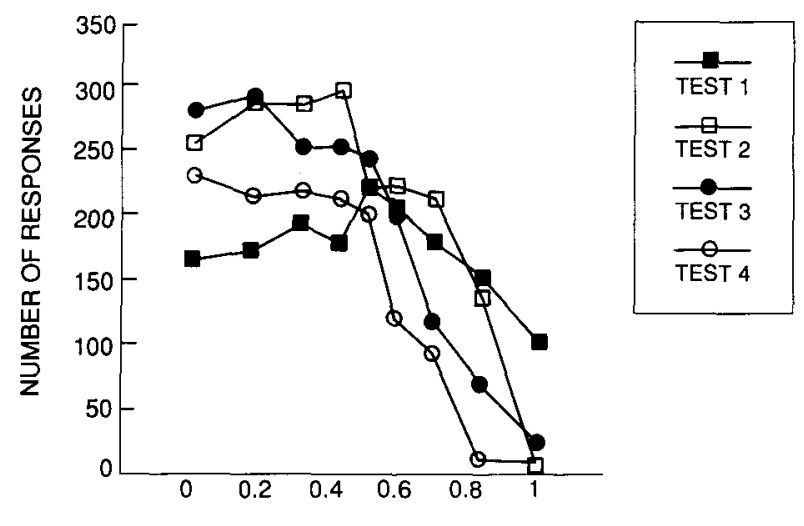

PROPORTION OF RED OR BLUE ELEMENTS

Figure 1. Prediscrimination and postdiscrimination gradients obtained in Experiments 1A-1C. The text describes the training conditions that preceded each test.
Table 1

Slope Indices and Response Measures From Experiment 1A-1C

\begin{tabular}{|c|c|c|c|c|c|c|}
\hline Subject & DR & $\begin{array}{c}\text { Total } \\
\text { Responses }\end{array}$ & + SI & Max & $-\mathrm{SI}$ & $\mathrm{Mi}$ \\
\hline \multicolumn{7}{|c|}{$\begin{array}{l}\text { Generalization Test } 1 \\
\text { (Baseline) }\end{array}$} \\
\hline 4947 & & 824 & .97 & 1.34 & 1.04 & .69 \\
\hline 4181 & & 1,587 & 1.14 & 1.40 & .78 & .71 \\
\hline 4568 & & 1,800 & .94 & 1.07 & .83 & .80 \\
\hline 232 & & 2,043 & .53 & .74 & .51 & .00 \\
\hline$M$ & & 1,564 & .90 & 1.14 & .79 & .55 \\
\hline \multicolumn{7}{|c|}{$\begin{array}{l}\text { Generalization Test } 2 \\
\text { (Simplified) }\end{array}$} \\
\hline 4947 & .93 & 2,053 & 1.84 & 2.44 & .68 & .06 \\
\hline 4181 & .90 & 1,871 & 1.18 & 1.25 & .53 & .02 \\
\hline 4568 & .88 & 2,479 & 1.14 & 1.44 & .69 & .00 \\
\hline 232 & 1.00 & 1,273 & 1.04 & 1.43 & .74 & .00 \\
\hline$M$ & .93 & 1,919 & 1.30 & 1.64 & .66 & .02 \\
\hline \multicolumn{7}{|c|}{$\begin{array}{c}\text { Generalization Test } 3 \\
\text { (Difficult) }\end{array}$} \\
\hline 4947 & .57 & 988 & .86 & 1.05 & .35 & .24 \\
\hline 4181 & .61 & 2,102 & 2.02 & 2.35 & 1.02 & .33 \\
\hline 4568 & .94 & 2,685 & .89 & 1.08 & .16 & .00 \\
\hline 232 & .56 & 1,153 & 1.13 & 1.80 & .80 & .00 \\
\hline$M$ & .67 & 1,732 & 1.22 & 1.57 & .58 & .14 \\
\hline \multicolumn{7}{|c|}{$\begin{array}{c}\text { Transfer Test } \\
\text { (64-Element Arrays) }\end{array}$} \\
\hline 4947 & .55 & 786 & .78 & .84 & .29 & .00 \\
\hline 4181 & .59 & 985 & 1.14 & 1.66 & .24 & .00 \\
\hline 4568 & .86 & 2,164 & 1.21 & 1.33 & .32 & 03 \\
\hline$M$ & .67 & 1,312 & 1.04 & 1.28 & .28 & .01 \\
\hline
\end{tabular}

Note-DR, discrimination ratio; SI, slope index; Max, maximum value; Min, minimum value.

tion ratios obtained from the last four training sessions before the postdiscrimination test.

During the second test, all 4 birds maintained an excellent discrimination between the two training values. Mean total responses to the positive array were 221 ; to the uniform negative array, they were 4 . Between these values, the patterns of responding varied. In general, the pigeons responded at rather high levels at least to the point at which there were 11 positive elements in the array, after which responding declined rather abruptly. Each bird also responded at high levels to the arrays on the positive side of $\mathrm{S}+$, containing more positive than negative elements. The positive slope index for each bird exceeded 1.0 (see Table 1). The maximum SI ranged from 1.25 to 2.44 . These data exemplify a peak shift.

\section{EXPERIMENT 1B Retraining and Testing With the Difficult Discrimination}

\section{Method}

In the next phase of research, we took advantage of the "easyto-hard effect." The same subjects were retrained with the original discrimination between the $50 \%$ mixture as $\mathrm{S}+$ and the patterns containing 11 positive and 25 negative elements as $S-$. The training procedure was the same as before. Only 1 bird mastered 
this discrimination, but all of them consistently responded more to the positive than to the negative arrays. Therefore, they were retested after various numbers of training sessions with the full range of proportions. The general procedures described previously remained in effect.

\section{Results}

In the discrimination between $S+$ and the patterns consisting of 25 negative elements, Subject 4568 attained a mean DR of .94 during the last 5 of 13 training sessions. Subject 232 attained a mean DR of .56 after 30 training sessions. The corresponding value for Subject 4947 was .57 after 33 sessions, and for Subject 4181 it was .61 after 12 training sessions.

During Test 3 , the birds again provided orderly discrimination gradients, rather similar to those obtained after the prior, easier discrimination. The mean gradient is shown in Figure 1. The negative wing of the gradient was understandably lower than before, since $S-$ was closer to $S+$ in the training procedure. Subject 4568 , the only bird to acquire the difficult discrimination, provided by far the steepest gradient among the negative values, as indicated by the slope indices in Table 1 . The measures of peak shift were very similar for the postdiscrimination gradients following the two training procedures. Each bird responded more to at least one test value on the positive side of $\mathrm{S}+$ than it did to $\mathrm{S}+$. This was most marked for Subject 4181 , although this bird acquired the training discrimination less well than the others. Subject 232 also showed a large peak shift.

\section{EXPERIMENT 1C Transfer to 64-Element Arrays}

Prior research on relative numerosity discriminations (Honig 1991a, 1991b; Honig \& Stewart, 1989) indicated that these discriminations were based on the proportions of positive and negative elements in the arrays, rather than on specific numbers of either. Similar gradients of relative numerosity were obtained when the arrays differed with respect to the total numbers of elements. In the present experiment, we determined whether the peak shift would also transfer in this fashion.

\section{Method}

The same subjects were retrained on the difficult discrimination for 11 sessions. They were then tested for 2 sessions with arrays of 64 rather than 36 elements. The proportions of elements corresponded closely to those used with 36 elements: 64 red elements; 53 red, 11 blue; 44 red, 20 blue; 37 red, 27 blue; 32 red, 32 blue; 27 red, 37 blue; 20 red, 44 blue; 11 red, 53 blue; 64 blue. The tests were carried out for two sessions without reinforcement. Each session consisted of four randomized blocks of trials.

\section{Results}

During retraining, 3 birds maintained their prior level of discrimination between equal proportions of red and blue elements, and the proportion of 25 of one kind and 11 of the other. Subject 4568 maintained a DR of about .86 ; for Subject 4181 and for Subject 4947 , the DRs were between .55 and .60 . Subject 232 failed to respond during several training sessions with 36 elements, and the discrimination was erratic when this subject did respond. This bird also responded rather little and erratically during the final test. The results from this subject are therefore not included.

The postdiscrimination gradient obtained from the 64element test is shown in Figure 1 as Test 4. The gradient is similar to the prior one obtained with 36-element arrays, although the total response level was lower. This difference may reflect either the change in the stimulus pattern, or the effects of repeated testing. Two birds showed a peak shift; they responded more to arrays with more positive than negative elements than they did to the positive training array (see Table 1). The gradient between the $50 \%$ mixture and the uniform negative array was somewhat steeper than the gradient obtained with 36 elements. This could be due to the additional training that intervened between the two tests, to repeated testing, or to the elimination of Subject 232. In spite of these modest differences, we can conclude that the pigeons were responding on the basis of the proportions of the elements in the array.

These results support the suggestion that relative numerosity is discriminated on the basis of the proportions of the different elements. This is of some interest, because other dimensions on which the peak shift has been obtained, such as spectral wavelength (Hanson, 1959) and auditory frequency (Jenkins \& Harrison, 1960), provide qualitative rather than quantitative differences among the stimuli to be discriminated.

\section{EXPERIMENT 2 Discrimination Training With a Range of Negative Values}

Experiment 2 was a systematic replication of Experiments $1 \mathrm{~A}-1 \mathrm{C}$, involving a maintained discrimination as the training procedure. The positive value was again the $50 \%$ mixture of red and blue elements. The negative values were the four proportions in which elements of one of the colors (red or blue) outnumbered the elements of the other color (blue or red). Accordingly, the birds were trained with one positive value (the $50 \%$ mixture) and four negative values. With this procedure, discrimination gradients could be obtained throughout training within the range of negative arrays. The effects of this procedure were then observed in two generalization tests with the full range of numerosity values. The first test was carried out with 36-element arrays, and the second was a transfer test with 64-element arrays.

\section{Method}

Four new pigeons were used for this experiment. The apparatus and the stimulus patterns were the same as those used for Experiments $1 \mathrm{~A}-1 \mathrm{C}$, as were the general procedures.

After initial training, each subject was run for four sessions with the positive stimulus patterns containing 18 elements of each color. Each session consisted of 24 reinforced trials. Discrimination train- 


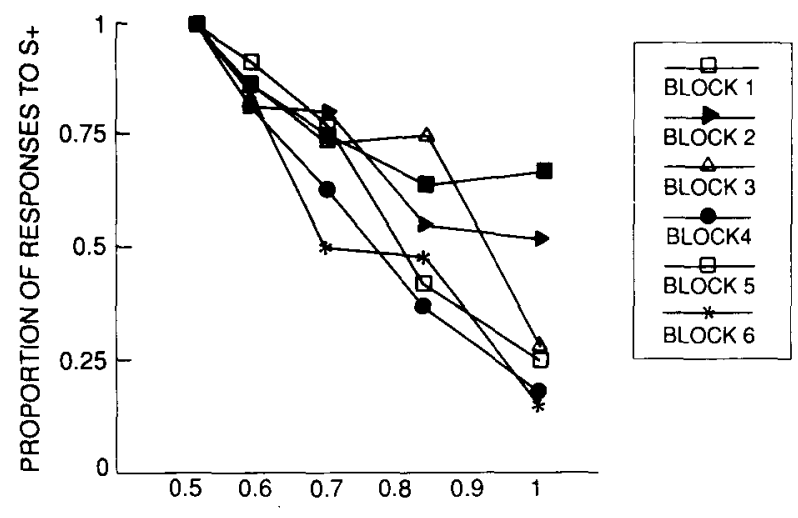

PROPORTION OF NEGATIVE ELEMENTS

Figure 2. Acquisition of the maintained discrimination in Experiment 2. There were four training sessions in each block.

ing followed. Each session consisted of four blocks of 6 trials. The positive proportion of elements was presented twice in each block, and each of the four negative proportions was presented once. For 2 birds, the negative proportions were the patterns that contained $21,25,30$, or 36 blue elements; for the 2 other birds, the negative patterns contained the same numbers of red elements.

All subjects were trained for four blocks of four sessions. At this time, reliable declining gradients of responding were obtained among the negative patterns, and the birds were tested in extinction for two sessions with the full range of arrays of 36 elements, as described in the account of Experiment 1A. Each session consisted of four blocks of nine trials each, presented in a randomized order.

The birds were then retrained for two more blocks of four sessions each, and then retested in extinction during two sessions with the transfer patterns of 64 elements, as described for Experiment $1 \mathrm{C}$.

\section{Results}

Discrimination training. All birds acquired the maintained discrimination between the $50 \%$ mixture and the patterns in which the numbers of one element exceeded the other. The mean data obtained from the six blocks of training sessions are shown in Figure 2. The birds acquired the discrimination in an orderly fashion. By the fourth block of sessions, prior to the first test, the discrimination was quite stable, with a DR for the uniform negative arrays of .18. After six blocks of training, this ratio was .15 . Responding was related to the proportion of positive elements roughly in a linear way, and each bird provided a decremental gradient. However, the graph of the mean data obscures some individual differences. Subject 4684 was not responding at all to the uniform negative pattern at the end of training, whereas Subject 757 was still responding to this pattern at about half of the rate at which it responded to the positive arrays. The negative slope indices shown in Table 2 reflect these differences.

Test results. The results from the generalization tests are shown as total response gradients in Figure 3. The mean gradient from the first test shows a marked peak shift within the range of positive values, and a decline beyond the shifted peak. Each subject responded more to arrays containing 21,25 , and 30 positive elements than to those containing 18 positive elements (the training $S+$ ). For 3 birds, responding declined beyond the shifted peak, but Subject 4684 actually responded most to the uniform array of positive elements. Three birds also discriminated very well among the negative arrays during the tests, but Subject 757 did not. (This was quite in accordance with the training data from this bird.) It is of some interest that this subject also showed less of a peak shift than the others.

The data obtained from the transfer test with 64 elements are also shown in Figure 3. On the whole, they are similar to those obtained with 36 elements. The peak of the transfer gradient is somewhat below the value obtained from the first test. Otherwise, the numbers of responses obtained during the tests were remarkably similar. Again, transfer between 36-element and 64-element arrays was based on the relative numerosities of the two elements.

Relevant data from the training procedure have been added to Figure 3. The mean data from the sixth block of four training sessions, which preceded the 64-element test, were rescaled so that the value for $\mathbf{S}+$ coincided with

Table 2

Slope Indices and Response Measures From Experiment 2

\begin{tabular}{|c|c|c|c|c|c|c|}
\hline Subject & $\begin{array}{l}\text { Discrimination } \\
\text { Training }\end{array}$ & $\begin{array}{c}\text { Total } \\
\text { Responses }\end{array}$ & $+\mathrm{SI}$ & $\operatorname{Max}$ & $-S I$ & \\
\hline \multicolumn{7}{|c|}{ Generalization Test } \\
\hline 757 & .80 & 952 & 1.34 & 1.71 & .94 & \\
\hline 4660 & .53 & 987 & 1.32 & 2.13 & .41 & \\
\hline 4684 & .20 & 1,663 & 1.50 & 1.73 & .18 & \\
\hline 45 & .46 & 301 & .84 & 1.38 & .36 & .02 \\
\hline$M$ & .50 & 976 & 1.25 & 1.74 & .47 & .25 \\
\hline \multicolumn{7}{|c|}{ Transfer Test } \\
\hline 757 & .64 & 985 & 1.10 & 1.43 & .77 & .69 \\
\hline 4660 & .56 & 526 & 1.02 & 2.04 & .40 & \\
\hline 4684 & .34 & 1,811 & 1.56 & 1.75 & .32 & .0 \\
\hline 45 & .44 & 341 & 2.30 & 3.81 & .73 & \\
\hline$M$ & .50 & 916 & 1.50 & 2.26 & .56 & .19 \\
\hline
\end{tabular}

Note-SI, slope index; Max, maximum value; Min, minimum value.

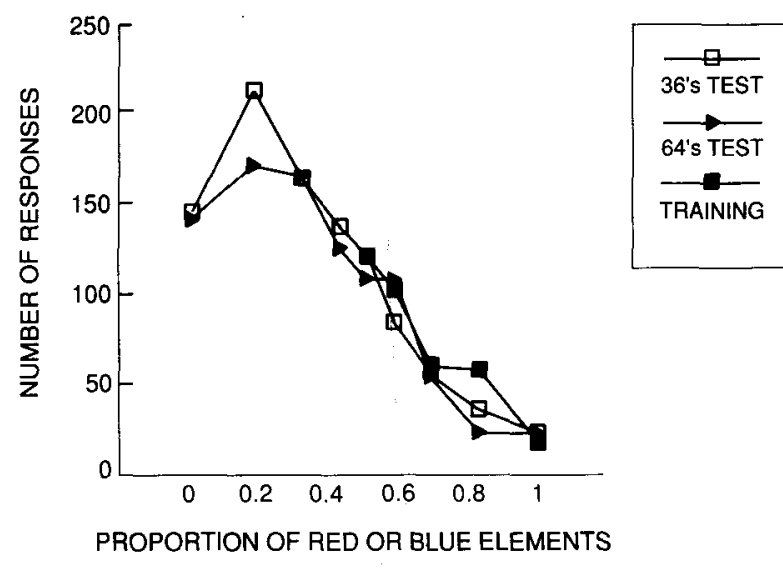

Figure 3. Postdiscrimination gradients obtained in Experiment 2, together with data from the last block of discrimination training sessions. The latter have been rescaled so that the value obtained from $S+$ in training coincides with the value obtained from $S+$ in testing. 
the number of responses obtained with that stimulus during that test. The other training values represent appropriate proportions of that adjusted value. When the data are compared in this way, the final discrimination gradient is very similar to the gradient obtained from the same values in testing. The elevated responding, obtained during testing with the positive values, did not induce negative contrast in the form of a reduction in responding among the training values.

The positive and negative slope indices are shown for each bird in Table 2. For 3 birds, the gradient obtained with 36 elements (the generalization test) provided a positive index greater than 1.0 , but even the remaining subject produced a shifted peak. Subject 757 had a marked elevated positive gradient, in spite of a poor discrimination of the negative proportions. The positive slope indices obtained from the transfer test with 64 elements (transfer test) are rather similar to those obtained from the prior test. If anything, they are higher, which may be due to the additional discrimination training carried out between the tests.

\section{Discussion}

This experiment provides a convincing replication of behavioral contrast and the peak shift, when a range of proportions of stimuli constituted the set of negative values. Orderly gradients of stimulus control were obtained during training from most of the birds. Even the 1 bird that did not discriminate well between the negative values and $\mathbf{S}+$ showed a peak shift during both tests. There was little reduction of responding during the test with the transfer arrays, even though the birds had not previously encountered the patterns of 64 elements. The eight additional sessions of discrimination training between the two tests may have counteracted an effect of a change in the stimulus patterns, but this seems unlikely. While the pigeons are very sensitive to the relative numerosities of the stimulus arrays, they seem not to discriminate between absolute numbers without special training procedures (Honig, 1993). In prior research on relative numerosity and stimulus mixture with small elements (Honig, 1991a; Honig \& Stewart, 1989), we also obtained good transfer of the discriminations among test arrays that differed in the absolute numbers of items.

\section{EXPERIMENT 3 Peak Shift With Different Orientations of Rectangles}

It is important to replicate the peak shift on the dimension of relative numerosity with a carrier dimension other than color. The pigeons may have been responding to the overall "blueness" (or "redness") of the arrays, rather than responding to the patterns as arrays of individual elements. Also, differences in color are often confounded with brightness and/or saturation. For the human observer, the red dots were somewhat brighter and more saturated than the blue dots. This is not a crucial matter for the interpretation of numerosity judgments, but a study that avoids such confounds with respect to the carrier dimension is certainly desirable.

This aim could be accomplished with arrays of small rectangles that differed only in orientation. For the human observer, arrays of vertical or horizontal rectangles did not look markedly "vertical" or "horizontal" in the way that arrays of blue or red elements looked markedly blue or red. The general training and test procedures were similar to those used in Experiment 2.

\section{Method}

Four new pigeons, maintained at about $80 \%$ of their free-feeding weights, were used for this study. Experimental events, including the stimulus displays, were controlled by a Zenith XT computer. The stimuli were presented on a 14-in. Zenith Flat Technology monitor, which was framed by a Carroll Touch infrared touch screen. The background color on the monitor was black. This apparatus provided considerable flexibility in the kinds of stimuli that could be displayed and in their arrangement. A response was registered whenever the pigeon's peck on the monitor broke perpendicular infrared beams from the touch screen, and the computer recorded the location of the peck according to the vertical and horizontal coordinates.

In the training and test procedures, 36 white rectangles were presented in a square matrix on each trial. The horizontal rectangles were $1.75 \mathrm{~cm}$ wide and $0.5 \mathrm{~cm}$ high; the vertical rectangles were $0.5 \mathrm{~cm}$ wide and $1.75 \mathrm{~cm}$ high. The rectangles were spaced $2 \mathrm{~cm}$ apart, center to center. The entire display was $16 \mathrm{~cm}$ on each side. The following proportions of rectangles were used for training and testing: 36 horizontal; 30 horizontal, 6 vertical; 24 horizontal, 12 vertical; 18 horizontal, 18 vertical; 12 horizontal, 24 vertical; 6 horizontal, 30 vertical; 36 vertical. The elements in the mixed arrays were randomly arranged by the computer on all trials in training and testing.

Procedure. As before, each positive trial lasted for $20 \mathrm{sec}$ before reinforcement became available. The first response after $20 \mathrm{sec}$ was reinforced, and the trial ended at that time. Negative trials ended after $20 \mathrm{sec}$. Each trial was followed by an intertrial interval of $10 \mathrm{sec}$, during which the screen was blank, and responses had no programmed consequences.

Preliminary training. The pigeons were trained to peck at arrays of elements in the following stages: A single white element, $1.0 \mathrm{~cm}$ square, appeared at various randomly selected locations during successive trials, and the pigeon had to peck at it. The pigeons then learned to peck at matrices of such squares, composed of 4 , 16 , and then 36 elements on successive days. Each session consisted of 20 trials.

Acquisition and testing with rectangles. During initial training, different randomized patterns of 18 horizontal and 18 vertical rectangles were presented during each session. There were 20 reinforced trials per session. After 15 sessions, a prediscrimination gradient was obtained during 2 sessions in which the various proportions of elements were presented. Each proportion was presented once in four randomized blocks of trials. No reinforcement was provided during these 20 -sec test trials. The test sessions were separated by 1 training session.

Discrimination training and postdiscrimination tests. The arrays with 18 rectangles of each kind were positive; the arrays with more horizontal than vertical elements, or the reverse (counterbalanced across subjects), were negative. Positive arrays were presented twice in each randomized block of trials, and the three negative proportions were presented once. Each training session comprised four blocks of trials.

After 20 sessions, 1 bird, Subject 1848, had learned the discrimination well enough to be tested. For the other 3 birds, each negative proportion was presented twice per block, rather than once, 
to enhance the discrimination. This had the desired effect, so that Subject 2151 and Subject 2122 could be tested after five further blocks of four training sessions, whereas Subject 482 was tested after six blocks.

The postdiscrimination test was carried out during two sessions, separated by one further training session. The test procedure was the same as that used to obtain the prediscrimination gradient.

\section{Results and Discussion}

The pigeons readily learned to peck at the initial training patterns, and they responded at stable rates to the arrays containing equal numbers of horizontal and vertical rectangles. The gradient from the prediscrimination test is shown in Figure 4. It provides little evidence for stimulus control based on initial acquisition or responding with equal numbers of elements. On the average, the birds responded 66 times to the training value of 18 vertical and 18 horizontal rectangles, which is only slightly more than the mean of responding to all of the test values (61 responses).

During the maintained discrimination procedure, responding to the uniform arrays declined reasonably quickly. During the third block of four sessions, the birds responded to the uniform arrays only one fifth as much as they did to positive arrays. Responding to the other negative proportions declined much less quickly for 3 of the 4 birds. As indicated above, individual birds required different numbers of training sessions to master the discrimination.

The mean data from the postdiscrimination test are shown in Figure 4, together with the mean discrimination gradient obtained from the last block of training sessions. The values from the latter have again been rescaled so that responding to the $50 \%$ mixture is shown at the same level as the test data obtained from the same array. The mean postdiscrimination gradient shows an orderly reduction in responding among the negative values, and a general elevation of responding among the positive test values. This provides a convincing peak shift, and there was no decline among the test patterns as the numbers of positive elements increased. The birds responded more to the "posi-

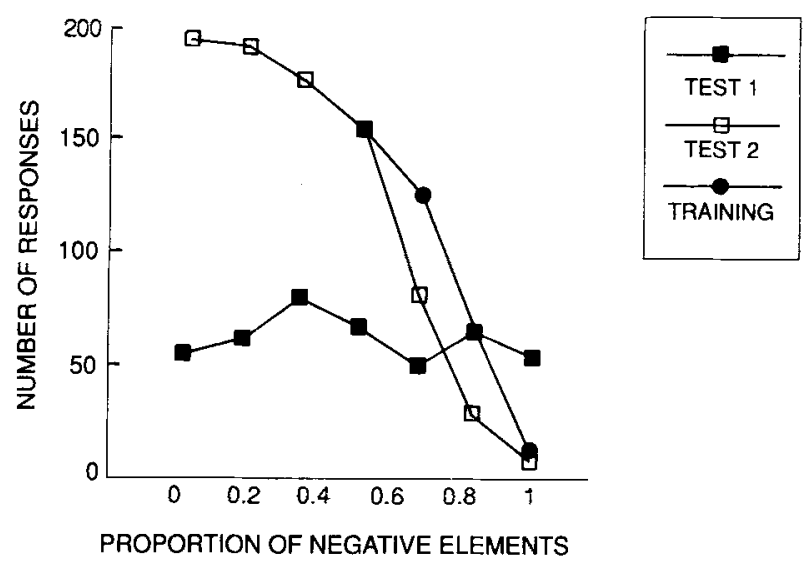

Figure 4. Prediscrimination (Test 1) and postdiscrimination (Test 2) gradients obtained in Experiment 3, together with data from the last block of training sessions. The latter have been rescaled as in Figure 3.
Table 3

Slope Indices and Response Measures From Experiment 3

\begin{tabular}{|c|c|c|c|c|c|c|}
\hline Subject & $\begin{array}{l}\text { Discrimination } \\
\text { Training }\end{array}$ & $\begin{array}{c}\text { Total } \\
\text { Responses }\end{array}$ & $+\mathrm{SI}$ & Max & $-S I$ & \\
\hline \multicolumn{7}{|c|}{ Prediscrimination Test } \\
\hline 2122 & & 360 & 1.03 & 1.28 & .90 & .9 \\
\hline 2151 & & 446 & .95 & 1.09 & .94 & .8 \\
\hline 482 & & 415 & 1.45 & 1.89 & 1.29 & .9 \\
\hline 1848 & & 481 & .80 & 1.11 & .52 & .4 \\
\hline$M$ & & 426 & 1.06 & 1.34 & .91 & .80 \\
\hline \multicolumn{7}{|c|}{ Postdiscrimination Text } \\
\hline 2122 & .38 & 1,004 & 1.06 & 1.13 & .20 & .01 \\
\hline 2151 & .52 & 697 & 1.11 & 1.33 & .30 & .09 \\
\hline 482 & .50 & 695 & 1.16 & 1.21 & .43 & .07 \\
\hline 1848 & .38 & 907 & 1.56 & 1.69 & .13 & .03 \\
\hline$M$ & .44 & 826 & 1.22 & 1.34 & .26 & .05 \\
\hline
\end{tabular}

Note-SI, slope index; Max, maximum value; Min, minimum value.

tive" test values than they did to the positive training value, with only two exceptions: Subjects 2151 and 2122 responded slightly less to the arrays containing 24 positive and 12 negative elements than they did to the arrays of 18 positive and 18 negative elements. In the remaining 10 comparisons between responses to the positive test proportions and positive training array, the birds responded more to the test arrays. The slope indices and other relevant data are shown for individual subjects in Table 3. These indices reflect the enhanced responding among positive test values after discrimination training.

As we found in Experiment 2, the gradient obtained from the negative training values was somewhat steeper in the postdiscrimination test than it was during the prior block of training sessions. This may again reflect negative contrast induced by the presentation of test values containing more than $50 \%$ positive elements, to which the birds responded at high rates.

This experiment shows that peak shift based on relative numerosity is not restricted to elements that differ in color. The areas of the small rectangles of different orientations can in principle be summated in each display by the pigeon's visual system, and the sums could be compared as a mechanism for determining relative numerosity. But this would be a more complex process than a summation of elements differing in color. The pigeon could summate the areas of blue and of red elements with no discrimination of other characteristics of the elements. A summation of the elements that have the same orientation requires an initial discrimination of specific features of the elements-namely, the relation of their horizontal and vertical dimensions. These features would presumably be lost once the areas were summated. A simpler interpretation is that the pigeons were able to discriminate directly the relative numerosities of the elements, which defined the dimension of stimulus control.

\section{GENERAL DISCUSSION}

Relative numerosity is a discriminable aspect of arrays of stimulus elements, both spatial patterns and temporal 
sequences. Prior research has provided orderly gradients on this dimension (Alsop \& Honig, 1991; Honig, 1991a, 1991b; Honig \& Stewart, 1989). The demonstration of a peak shift supports the dimensional nature of relative numerosity, as this provides a "convergent operation" (Hempel, 1952) for the identification of effective dimensions of stimulus control.

The peak shift was first obtained by Hanson (1959) on the spectral dimension. Steep gradients are normally obtained on this dimension from pigeons, even after simple acquisition with a single training value. By contrast, gradients of relative numerosity are relatively flat in the absence of explicit discrimination training between different proportions of elements. This is shown by the prediscrimination gradients obtained in Experiments $1 \mathrm{~A}$ and 3. The postdiscrimination gradients were also rather flat within the range of the positive proportions, so that there was little decrement "beyond" a shifted peak. This could again be due to the subject's lack of sensitivity to differences in relative numerosity. By contrast, colors are salient aspects of local features, including other birds, flowers, and so forth. With more extended aspects of the environment, sensitivity to the characteristics of the elements may be sacrificed in the interest of integrating information from a wider view.

Stimuli traditionally used for the study of stimulus control are characteristic of specific objects or events. Spectral values (Guttman \& Kalish, 1956) characterize the plumage of many birds and flowers. Auditory frequencies (Jenkins \& Harrison, 1960) determine the pitch of calls of various birds, and natural contours are distinguished by their orientations (Honig et al., 1963). The "sign stimuli" of ethology tend to be prominent, identifiable features carried or produced by an animal. Although such aspects of the environment are not as simple or uniform as the stimuli used in most experiments, they do not usually comprise arrays of discrete elements. The arrays used in our experiments are more representative of aggregations, clusters, or patterns of environmental features, such as groups of other birds or animals, trees, flowers, stones, and so forth. Such arrays provide cues for spatial localization, and they can determine the attractiveness of particular locations that provide necessities of life, such as food or nesting material, or the company (or avoidance) of conspecifics. If an animal has identified favorable and unfavorable characteristics of, say, different kinds of plants in its natural habitat, it will most likely choose clusters of plants that provide the highest proportion of the favorable ones, even if its initial experience was in a setting with a less favorable distribution. This would be a naturalistic counterpart to the peak shift.

The peak shift, when obtained from extended arrays of elements, supports the notion that derived dimensions of stimulus control such as relative numerosity are functionally similar to the dimensions that characterize single elements. Indeed, for the pigeon or for other animals, "fundamental" and "derived" dimensions of stimulus control may not be distinct categories. If a bird is searching for red berries within a patch, it will favor the reddest, and presumably the ripest, berries. If the berries are not randomly distributed-for example, the reddest berries may be more numerous on the sunny side of the bush - the bird will forage in the most favorable area. Thus, if the discrimination of relative numerosity provides an assay for the bird of the optimality of different settings or patterns, then peak shift as obtained in the present experiments is a reasonable outcome. It would simply reflect the total attractiveness of the positive elements in the array.

This functional account of peak shift differs from an explanation based on the stimulus dimension, which was suggested by Spence as early as 1937, in an attempt to explain transposition. He postulated that discrimination training generated a gradient of excitation around $\mathrm{S}+$ and a gradient of inhibition around S-. The algebraic summation of these gradients could yield postdiscrimination gradients with a shifted peak. Hanson's (1959) research supported this prediction; he obtained the peak shift with spectral values as the stimulus dimension, and this has been replicated many times thereafter (see Rilling, 1977, and Honig \& Urcuioli, 1981, for reviews). Although these findings supported Spence's analysis, behavioral contrast had to be invoked to explain the high levels of responding obtained during the postdiscrimination tests of stimulus control. A simple algebraic summation of positive and negative gradients, as proposed by Spence, did not provide a satisfactory model.

Spence's (1937) model predicted a decline of responding "beyond" the shifted peak. This has typically been obtained with pigeons on the spectral dimension-for example, by Hanson (1959), Honig (1962), and Honig et al. (1959). In Experiments 1A-1C, only the first of the three postdiscrimination gradients declined beyond the shifted peak, and that was only by a modest amount. In Experiment 2 , there was again a small decline beyond the shifted peak. In Experiment 3, the gradient increased even up to the uniform positive arrays.

The reason for this difference between our results and the prior findings is not clear. It may be due to the subjects' sensitivity to the stimulus dimensions. Pigeons discriminate readily among spectral values, and they provide decremental gradients even after simple acquisition. In the absence of any discrimination training, relative numerosity gradients tend to be rather flat, as shown in $\mathrm{Ex}$ periments $1 \mathrm{~A}-1 \mathrm{C}$ and 3 . Even the postdiscrimination gradients yielded a "plateau" rather than a "peak" among the "positive" arrays. This may simply reflect the nature of control by a derived stimulus dimension. The range of the available test values in our research may be short in relation to the size of the discriminable differences on this perceptual continuum.

However, there is another possible reason for our failure to obtain true peaks in our gradients. When individual stimuli define a stimulus dimension, they necessarily differ in appearance. For example, in the experiment by Hanson (1959), the positive stimulus of $550 \mathrm{~nm}$ was greener than the various negative values, ranging 
from 555 to $590 \mathrm{~nm}$, which shaded into yellow. The shorter wavelengths presented in testing became "less green" (and "more blue") at some point between 550 and $480 \mathrm{~nm}$. In the present research, the elements never changed in color or in orientation; only their distribution varied. Therefore, any increase in the proportion of positive elements would have made the array "more positive" and/or "less negative." Indeed, in Experiment 3, we observed a continued increase in responding with larger proportions of positive elements, up to the uniform positive array.

A choice procedure between different arrays may provide a more sensitive method for assessing the attractiveness of different arrays in testing. The pigeon may choose indifferently among the "positive" test arrays, reflecting the flatness of the postdiscrimination gradients in this range. On the other hand, it might consistently choose arrays with greater numbers of positive elements. In a study on the relation between peak shift and choice (Honig, 1962), pigeons chose between stimuli in accordance with the postdiscrimination gradients. This resulted in transposition between $\mathrm{S}+$ and the shifted peak, and reversal of transposition "beyond" the shifted peak. A corresponding relationship has not been determined for discrimination of relative numerosity. If choices between two arrays follow the simple principles identified by Honig, the pigeons should choose indifferently between the patterns in the "positive" range if the gradients are rather flat. But if the pigeon is sensitive to the proportions of positive and negative elements even within this range, it may choose differentially even between patterns that control similar rates of responding when they are presented separately.

The study of stimulus control has traditionally been carried out with stimuli that represent "abstractions from reality" in the form of line orientations, pure tones, spectral values, and so forth. Explicit discrimination procedures have rather similar effects with stimuli from several different dimensions. This permits psychologists to identify the general characteristics of stimulus control, and to speculate about the degree to which they apply to aspects of the environment. The present results were obtained with patterns of stimuli that were characteristic of environmental arrays rather than individual objects. This suggests that the familiar principles of stimulus control are valid well beyond the limited procedures with which they were discovered many years ago.

\section{REFERENCES}

Alsop, B., \& HoNIG, W. K. (1991). Sequential stimuli and relative numerosity in pigeons. Journal of Experimental Psychology: Animal Behavior Processes, 17, 386-395.

Guttman, N., \& Kalish, H. I. (1956). Discriminability and stimulus generalization. Journal of Experimental Psychology, 51, 79-88.

Hanson, H. M. (1959). Effects of discrimination training on stimulus generalization. Journal of Experimental Psychology, 58, 321-344.

HEMPE L, C. G. (1952). Fundamentals of concept formation in empirical science. Chicago: University of Chicago Press.

Honig, W. K. (1962). Prediction of preference, transposition and transposition-reversal from the generalization gradient. Journal of Experimental Psychology, 64, 239-248.

HoNIG, W. K. (1991a). Discriminability and distinctiveness in complex arrays of simple elements. In J. A. Nevin, M. C. Davison, \& M. L. Commons (Eds.), Quantitative analyses of behavior: Vol. 10. Signal detection: Mechanisms, models and applications (pp. 103-120). Hillsdale, NJ: Erlbaum.

HoNIG, W. K. (1991b). Discrimination by pigeons of mixture and uniformity in arrays of stimulus elements. Journal of Experimental Psychology: Animal Behavior Processes, 17, 68-80.

HoNIG, W. K. (1993). Numerosity as a dimension of stimulus control. In S. Boysen \& E. J. Capaldi (Eds.), The development of numerical competence: Animal and human models (pp. 61-86). Hillsdale, NJ: Erlbaum.

Honig, W. K., Boneau, C. A., Burstein, K. R., \& Pennypacker, H. S. (1963). Positive and negative generalization gradients obtained after equivalent training conditions. Journal of Comparative \& Physiological Psychology, 56, 111-116.

Honig, W. K. \& Stewart, K. E. (1989). Discrimination of relative numerosity by pigeons. Animal Learning \& Behavior, 17, 134-146.

Honig, W. K., Thomas, D. R., \& Guttman, N. (1959). Differential effects of continuous extinction and discrimination training on the generalization gradient. Journal of Experimental Psychology, 58, 145-152.

Honig, W. K., \& URCuioli, P. J. (1981). The legacy of Guttman and Kalish (1956): Twenty-five years of stimulus generalization research. Journal of the Experimental Analysis of Behavior, 36, 405-445.

JeNkins, H. M., \& HARRISON, R. H. (1960). Effect of discrimination training on auditory generalization. Joumal of Experimental Psychology, 59, 246-253.

RILLING, M. (1977). Stimulus control and inhibitory processes. In W. K. Honig \& J. E. R. Staddon (Eds.), Handbook of operant behavior (pp. 432-480). Englewood Cliffs, NJ: Prentice-Hall.

SPENCE, K. W. (1937). The differential response of animals to stimuli differing within a single dimension. Psychological Review, 44, 430-444.

(Manuscript received May 28, 1992;

revision accepted for publication February 18, 1993.) 